\title{
Vector tomography for the coronal magnetic field
}

\section{Longitudinal Zeeman effect measurements}

\author{
M. Kramar, B. Inhester, and S. K. Solanki \\ Max-Planck-Institut für Sonnensystemforschung, 37191 Katlenburg-Lindau, Germany \\ e-mail: [kramar;inhester; solanki] @mps .mpg.de \\ Received 16 January 2006 / Accepted 13 April 2006 \\ ABSTRACT

\begin{abstract}
Aims. We study the possibility of applying tomographic techniques in order to reconstruct the 3D magnetic field configuration in the solar corona. Our simulations are based on data which can be obtained from longitudinal Zeeman-effect (or from Faraday rotation) measurements obtained during a solar rotation.

Methods. The Zeeman effect provides essentially the integrated line-of-sight component of the magnetic field. The reconstruction problem relates to a family of similar problems termed vector tomography. For inversion of this type problems it is known that the curl-free part of a vector field cannot be reconstructed from the integrated along line-of-sight data. To remove the resulting ambiguity of the reconstruction we include the additional constraint, $\nabla \cdot \boldsymbol{B}=0$, into the inversion similar to smoothing constraints in Tikhonov regularization problems.

Results. It is shown that Zeeman data obtained from vantage points in the ecliptic plane alone is sensitive only to certain magnetic field structures. For a full reconstruction it is necessary either to have also observations from viewing directions at higher heliographic latitudes, or to combine the longitudinal Zeeman-effect data with observations that provide the transverse component of the field, e.g. from the Hanle effect.
\end{abstract}

Key words. Sun: corona - Sun: magnetic fields - Sun: infrared - polarization

\section{Introduction}

The magnetic field of the solar corona plays a key role in both static and dynamic processes in the corona, e.g., formation of loops and filaments, coronal heating, solar flares and filament eruptions. A great obstacle in our understanding of these processes is our inability to properly determine this magnetic field.

The conventional way to estimate the coronal field is by extrapolation from measurements of the line-of-sight field component at the solar surface using the potential field approximation. But the potential field extrapolation does not take into account any electric current in the corona and, therefore, misses an important part of the physics. Since the potential magnetic field is the field with the minimum energy for a given photospheric radial boundary condition (Sakurai 1989), it cannot account for dynamical processes such as eruptions and flares, where very probably magnetic energy is converted into plasma kinetic energy without a marked change in the boundary flux on the same fast time scale. Indeed, soft X-ray observations of active regions often show a non-potential structure of the magnetic field (Jiao 1997). Recently, vector magnetograms from the photosphere have become available which in principle supply all the information necessary for a non-linear force-free field extrapolation of the surface data (Wiegelmann 2005). The more realistic force-free field extrapolation, however, is highly ill-posed and yields less reliable results, the larger the distance from the surface and the stronger the currents (Demoulin 1992).

Direct, though line-of-sight (LOS) integrated information about the coronal magnetic field can be obtained from the
Zeeman and the Hanle effects applied to coronagraphically observed lines of highly ionized species. In the past most such information has been obtained by observations of resonantly scattered coronal lines above the limb, e.g., the coronal emission lines at $\lambda=530.3 \mathrm{~nm}$ and $\lambda=1074.7 \mathrm{~nm}$ scattered by Fe XIV and Fe XIII, respectively (House et al. 1982; Querfeld 1982; Arnaud 1982; Arnaud \& Newkirk 1987). For these "forbidden" lines, the life time in the excited state is much longer than the Larmor period, conditions usually referred to as the "strong field" regime. The method makes use of the Stokes- $Q$ and $-U$ components (linear polarization) of the line produced by resonant scattering modified by the Hanle effect. The polarization plane of the scattered line radiation is for "strong field" conditions related to the orientation of the magnetic field in the plane normal to the LOS. The method was first investigated by Charvin (1965) and later developed in more detail by Sahal-Brechot (1974a,b, 1977), House (1977), Casini \& Judge (1999, 2000), Raouafi et al. (2002), Landi Degl'Innocenti \& Landolfi (2004) and many others authors.

Recently, promising measurements of the longitudinal Zeeman effect have been achieved for infrared coronal lines (Judge 1998; Lin et al. 2000). In the infrared the ratio between the Zeeman splitting and the line width is more favorable than in the visible. Lin et al. $(2000,2004)$ could even determine both the line-of-sight magnetic flux density from the Stokes- $V$ and and the transverse field orientation from an observation of the Hanle effect in the Stokes- $Q$ and- $U$ components of the Fe XIII line at $\lambda=1075 \mathrm{~nm}$. Even though these measurements were restricted to heights below about $0.45 R_{\odot}$ above an active region, 
they demonstrate that the measurement technique has the potential for scanning the whole corona on a regular basis with a sensitivity of about $1 \mathrm{G}$.

The interpretation of the results of both the Zeeman and Hanle measurements, however, is complicated by the fact that they are obtained above the limb. Thus they represent quantities that are LOS integrated through an optically thin corona. Changes in the polarity of the LOS component or of the direction of the transverse component of the field along the LOS can strongly reduce the value of such measurements if the structure along the LOS cannot be disentangled. In this and a followup paper we therefore investigate whether these measurements can be used to reconstruct a valid model of the 3D coronal field distribution and thereby overcome the shortcomings of the raw measurements and pure photospheric field extrapolation.

The inversion of LOS data to a 3D distribution usually is referred to as tomographic inversion. Unlike the conventional scalar tomography (Natterer 1986), we here aim to reconstruct a vector field and therefore have to employ methods of vector tomography (Sparr \& Strahlen 1998), an extension of ordinary scalar tomography. The fields which can be reconstructed depend strongly on the component observed along the line of sight. We will therefore treat the reconstruction of longitudinal Zeeman or Faraday data and essentially transverse Hanle data separately. This paper is devoted to Zeeman data, a similar study for saturated Hanle data will be given in a follow-up paper.

The plan of the present paper is as follows. In Sect. 2 we discuss the integration kernel for longitudinal Zeeman data which is needed to properly interpret the observations quantitatively. In Sect. 3 we briefly outline some basics of vector tomography and define the inversion problem we have to deal with. The inversion method and test results are given in Sects. 4 and 5, respectively. A discussion in the light of present experimental restrictions is given in the final section.

\section{Integration kernel for longitudinal Zeeman data}

The physics of line formation in the presence of a magnetic field, in particular the Zeeman effect, has been widely described in the literature (see the textbooks Sobel'man 1972; Stenflo 1994; Thorne et al. 1999; Landi Degl'Innocenti \& Landolfi 2004). We reiterate here only the key points of the effect which are essential for the coronal plasma and which are needed to derive the tomography inversion formulae.

The ratio of the Zeeman splitting to the thermal line width in the coronal plasma is very small due to the small field strength $(\sim 10 \mathrm{G})$ and the large thermal broadening $\left(10^{6} \mathrm{~K}\right)$. Consequently, a first order Taylor expansion of the Zeemanshifted left and right hand polarized line profiles can be used to express the emission coefficients for the Stokes- $I$ and- $V$ components emitted during a dipole transition of atomic states $\left(\alpha_{0} J_{1}\right) \rightarrow\left(\alpha_{0} J_{0}\right)$. For dipole transitions, $|\Delta J|=1$ and $\alpha_{0}$ stands for the quantum numbers of the the electronic ground state except for the total angular momentum $J$. The expressions for the local emissivity are (Casini \& Judge 1999, 2000)

$$
\begin{aligned}
& \varepsilon_{I}\left(\omega, \boldsymbol{r}, \hat{\boldsymbol{e}}_{\mathrm{LOS}}\right)=\frac{\hbar \omega}{4 \pi} N_{\alpha_{0} J_{1}} A \phi(\omega)\left(1+D \sigma_{0}^{2} \frac{3 \cos ^{2} \theta-1}{2^{3 / 2}}\right) \\
& \varepsilon_{V}\left(\omega, \boldsymbol{r}, \hat{\boldsymbol{e}}_{\mathrm{LOS}}\right)=-\frac{\hbar \omega}{4 \pi} N_{\alpha_{0} J_{1}} A \omega_{\mathrm{L}} \cos \theta \frac{\mathrm{d} \phi(\omega)}{\mathrm{d} \omega}\left(\bar{g}+E \sigma_{0}^{2}\right) .
\end{aligned}
$$

The coefficients in these expressions which depend on spatial coordinates either via their dependence on $\boldsymbol{B}$ or in another manner are the Larmor frequency $\omega_{\mathrm{L}}$, the local angle $\theta$ between $\boldsymbol{B}$ and the direction to the observer defined by the unit vector $\hat{\boldsymbol{e}}_{\mathrm{LOS}}$, the population density $N_{\alpha_{0} J_{1}}$ of the excited electronic state of the line transition and the alignment factor $\sigma_{0}^{2}$. The remaining coefficients are purely atomic constants like the Einstein coefficient $A$ for the spontaneous recombination for the transition and the effective Landé factor $\bar{g}$ of the transition. The factors $D$ and $E$ are dimensionless functions of the angular moment $J_{1}$ and $J_{0}$ and are of the order of unity. The spectral variation of the line is usually expressed through the Voigt profile function $\phi(\omega)$ in terms of the observing wave frequency $\omega=2 \pi c / \lambda$.

The coefficients which have direct or indirect spatial dependence are the more important for the inversion of the data, like, e.g., the population density of the excited level $N_{\alpha_{0} J}$ which depends on the local plasma density, temperature and the local intensity of the primary solar radiation. The alignment factor, $\sigma_{0}^{2}$, characterizes the inequality in population of the $\pi$ and $\sigma$ magnetic sublevels of the excited $J_{1}$ state. It depends on the distance from the Sun and also through the factor $\left(3 \cos ^{2} \gamma-1\right)$ on the angle $\gamma$ between $\boldsymbol{B}$ and $\hat{\boldsymbol{e}}_{\text {Sun. }}$. Here $\hat{\boldsymbol{e}}_{\text {Sun }}$ is the unit vector directed from the center of the Sun to the observing volume. The absolute value of $\sigma_{0}^{2}$ varies between values $\ll 1$ close to the Sun where the solar radiation is almost isotropic to a value of $1 / \sqrt{2}$ at large distances. A more detailed description and ways of calculating the $\sigma_{0}^{2}$, as well as other parameters involved in (1)-(2), can be found in Casini \& Judge (1999, 2000) and Landi Degl'Innocenti \& Landolfi (2004).

From (1)-(2) one can obtain magnetograph formula (Casini \& Judge 1999, 2000):

$\varepsilon_{V}\left(\omega, \boldsymbol{r}, \hat{\boldsymbol{e}}_{\mathrm{LOS}}\right)=k \bar{g} \frac{\partial \varepsilon_{I}(\omega, \boldsymbol{r})}{\partial \omega} \omega_{\mathrm{L}} \cos \theta$

where the correction factor $k$ describes the influence of a finite $\sigma_{0}^{2}$ on $\varepsilon_{V}$ in (3):

$$
k=\frac{1+\sigma_{0}^{2} E / \bar{g}}{1+\sigma_{0}^{2} D\left(3 \cos ^{2} \theta-1\right) /(2 \sqrt{2})}
$$

For the $J=1 \rightarrow 0$ transition, the maximal value of $k$ is 2 in case of no collisions and maximum anisotropy of the incident radiation. In the other limit of completely isotropic incident radiation, $\sigma_{0}^{2} \rightarrow 0$ and as consequence $k=1$. In the corona, especially in the lower corona, we have partially anisotropic incident radiation and isotropic collisions which reduce value of $\sigma_{0}^{2}$, and the values of $k$ are distributed near unity. How wide this distribution is depends on the magnetic field configuration, anisotropy factor and collisions. In this study, we will restrict the investigated coronal volume to a height below one solar radius where most of the Fe XIII emission comes from and $\sigma_{0}^{2}$ stays well below its maximum value. So, in the remainder of this paper we will assume that $\sigma_{0}^{2}$ vanishes in (1)-(4), and therefore take $k=1$. Finally, this assumption amounts to linearizing the tomographic inversion problem because in (3) the magnetic field now only enters through $\omega_{\mathrm{L}} \cos \theta$. The full, weakly nonlinear inversion problem will be investigated later. We are convinced that this linearization does not fundamentally change the nature of the inversion problem.

With assumption of vanishing alignment factor and ignoring instrument dependent coefficients and sensitivities, the LOS 


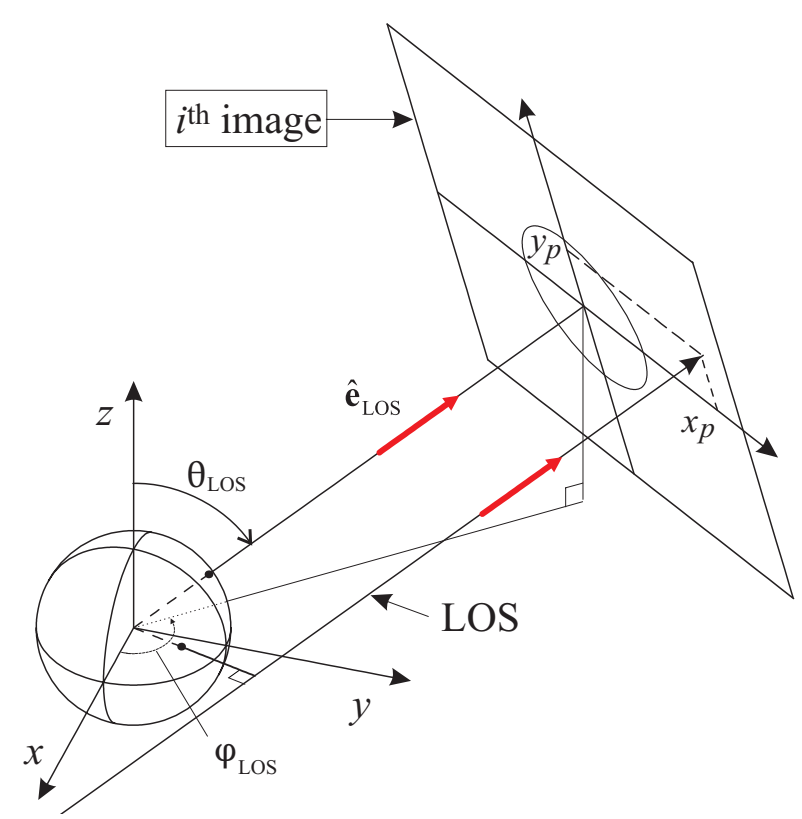

Fig. 1. The 3D view of the tomography geometry. $(x, y, z)$ is the reference system fixed to the Sun. The results of the reconstruction are presented in this reference system. $\left(x_{p}, y_{p}\right)$ is the reference system individual to every image in which the image data is defined. The LOS direction, $\hat{\boldsymbol{e}}_{\mathrm{LOS}}$, are defined by the angles $\varphi_{\mathrm{LOS}}$ and $\theta_{\mathrm{LOS}}$.

integrated Stokes- $I$ and Stokes- $V$ signals for an image $i$ and image pixel $p$ (Fig. 1) can be written as

$$
\begin{aligned}
I_{p, i} & =\frac{\hbar \omega}{4 \pi R^{2}} A \int_{\operatorname{LOS}_{p, i}} N_{\alpha_{0} J_{1}} \phi(\omega) \mathrm{d} \ell=\frac{1}{R^{2}} \int_{\operatorname{LOS}_{p, i}} \varepsilon_{I}(\omega, \boldsymbol{r}) \mathrm{d} \ell \\
V_{p, i} & =\frac{\bar{g}}{R^{2}} \int_{\operatorname{LOS}_{p, i}} \frac{\partial \varepsilon_{I}(\omega, \boldsymbol{r})}{\partial \omega} \omega_{\mathrm{L}} \cos \theta \mathrm{d} \ell \\
& =\frac{1}{R^{2}} \int_{\operatorname{LOS}_{p, i}} K(\omega, \boldsymbol{r})\left(\boldsymbol{B} \cdot \hat{\boldsymbol{e}}_{\mathrm{LOS}}\right) \mathrm{d} \ell .
\end{aligned}
$$

Here, $R \sim 1 \mathrm{AU}$ is the distance to the observer. We will refer to

$K(\boldsymbol{r}, \omega)=\frac{e \bar{g}}{m} \frac{\partial \varepsilon_{I}(\omega, \boldsymbol{r})}{\partial \omega}$

as the integration kernel. As pointed out above, its major spatial variation stems from the rapidly decreasing plasma density and hence from the population density $N_{\alpha J_{1}}$. A finite alignment factor will slightly modify the spatial variation and introduce a mild dependence of $K$ on the Van Vleck factor and hence on the magnetic field direction. Note that index $p$ refers to different spatial locations of image pixels, while index $i$ refers to different times of observation, i.e. to different observation angles.

An integrand similar to (6) is obtained also for observations of the Faraday rotation of the polarization plane (Stelzried et al. 1970). In this case, the kernel $K$ is directly proportional to the electron density. The practical disadvantage of these observations is that they usually cover only a small part of the plane of the sky.

With the assumption $\sigma_{0}^{2} \ll 1$, the value of $\varepsilon_{I}$ does not depend on the magnetic field, and we can in principle invert (5) for $\varepsilon_{I}(\omega, \boldsymbol{r})$ first by means of ordinary scalar tomography applied to Stokes- $I$ intensity line profile observations at different values of $\omega$. If we rather invert $\partial I_{p, i} / \partial \omega$, we obtain directly $\partial \varepsilon(\omega, \boldsymbol{r}) / \partial \omega$ needed for the kernel (7). We can therefore assume that the kernel $K(\boldsymbol{r}, \omega)$ is known. The nice property of using the magnetograph formula (3), in contrast to the Faraday-effect (in this case kernel $K$ is the electron density), is that it is therefore not necessary to assume a model distribution of the ion density, temperature and plasma velocity over the corona to find the respective kernel $K(\boldsymbol{r}, \omega)$. In practice, however, the influence of stray light will have to be carefully accounted for.

In general, (6) depends on wavelength and the data from a whole line scan should be used. For the test calculations presented here, we assume that data for a single wavelength is used only and we omit the wavelength dependence in the following. The spatial variation of $K$ for a single wavelength depends largely on the density variation. However, the spatially varying width of $\phi(\omega)$ due to a variable coronal temperature or shifts due to a finite plasma motion may also be folded in.

If the above approximation of a vanishingly small alignment factor had not been made, we would have retained some magnetic field dependence in $\varepsilon_{I}$ and $K$. Provided the factors $\sigma_{0}^{2}, D, E$ in (1) and (2) are known, we then could not solve (5) and (6) successively anymore but would have to solve them simultaneously. Moreover, in this case it is necessary to calculate the alignment factor using statistical equilibrium equations for the populations of all levels involved in the formation of the line. We believe that the conditions for solvability for the magnetic field of this more complicated system do not greatly differ from the approximate system investigated here as the principal problem is the limitation of the observations to the LOS component of $\boldsymbol{B}$ in (6).

\section{Basics of vector tomography}

Scalar tomography has been applied in solar physics for the reconstruction of the coronal electron density (Davila \& Thompson 1992; Davila 1994; Zidowitz 1997, 1999). It uses measurements of the LOS integral of white light scattered by coronal free electrons (Thomson-scattering). For the inversion, a rigid rotation of the coronal density structures with the Sun about the ecliptic normal is usually assumed. Coronagraph data from half a solar rotation then is necessary as input. As a consequence, only structures which are stationary over about 14 days can reliably be reconstructed. A detailed description of scalar tomography can be found in Natterer (1986), including a description of the "exterior tomography problem" which is typical for coronagraph data. This data have a gap in its center, which for coronagraph data is due to the occultation of the Sun.

In the case of vector tomography, the scalar in the integrand of the LOS integral is replaced by a function which depends on some component of the vector field $\boldsymbol{B}$ to be reconstructed. The essential complication introduced here is that this field component depends explicitly on the LOS direction $\hat{\boldsymbol{e}}_{\text {LOS }}$. Hence the contribution a volume element makes to an observation now depends explicitly on the direction it is looked at. This is an essential difference with respect to scalar tomography where the scalar integrand does not depend on the view direction. Vector tomography has been applied for the reconstruction of fluid flows in ordinary fluids and plasmas (Sparr \& Strahlen 1998; Osman \& Prince 1998) but not to our knowledge to the reconstruction of a magnetic field.

We argued in the previous section that for the Zeeman and Faraday effect observations the data obtained during the measurements at pixel $p$ in image $i$ can within the assumptions described above be expressed in the form (6) where we can assume that the kernel $K$ is a known function of $\boldsymbol{r}$. 
A fundamental limitation of vector tomography has been pointed out by Norton (1989): with observations of the type (6) and in the case $K=$ const. only the divergence-free part of $\boldsymbol{B}$ can be reconstructed. We may combine $K(\boldsymbol{r}) \cdot \boldsymbol{B}(\boldsymbol{r})$ to a new field $\boldsymbol{V}(\boldsymbol{r})$ so that (6) becomes

$$
V_{p, i}=\int_{\text {LOS of } p, i}\left(\boldsymbol{V} \cdot \hat{\boldsymbol{e}}_{\mathrm{LOS}}\right) \mathrm{d} \ell .
$$

We then may Helmholtz-decompose $\boldsymbol{V}$ into an incompressible part $\boldsymbol{V}^{\prime}$ and a potential field

$\boldsymbol{V}(\boldsymbol{r})=\boldsymbol{V}^{\prime}(\boldsymbol{r})-\nabla \phi(\boldsymbol{r}), \quad \nabla \cdot \boldsymbol{V}^{\prime}=0$.

Insertion into (8) yields

$$
\begin{aligned}
V_{p, i} & =\int_{\operatorname{LOS} \text { of } p, i}\left[\left(\hat{\boldsymbol{e}}_{\operatorname{LOS}(p, i)} \cdot \boldsymbol{V}^{\prime}\right)-\left(\hat{\boldsymbol{e}}_{\operatorname{LOS}(p, i)} \cdot \nabla\right) \phi\right] \mathrm{d} \ell \\
& =\int_{\operatorname{LOS} \text { of } p, i}\left(\hat{\boldsymbol{e}}_{\operatorname{LOS}(p, i)} \cdot \boldsymbol{V}^{\prime}\right) \mathrm{d} \ell-\left.\phi\right|_{\ell=-\infty} ^{\ell=+\infty} .
\end{aligned}
$$

This reveals that $V_{p, i}$ does not depend on the details of $\phi$ and hence on the potential part of $\boldsymbol{V}$ along the LOS.

Therefore with data which are only sensitive to the LOS component of a vector field we are unable to resolve the curl-free part of the field $\boldsymbol{V}$, independent of how good the resolution of our instrument (number of indices $p$ ) or how densely we choose the sampling of the view directions (number of indices $i$ ). The space of curl-free vector fields therefore represents a null space to our inversion problem. A similarly fundamental null space does not exist in scalar tomography.

The presence of this null space is also one of the reasons why vector tomography is usually applied to incompressible flow fields for which the contribution from this null space is known to vanish a-priori. For the field $\boldsymbol{V}=K \boldsymbol{B}$ we cannot expect incompressibility to hold, but we can make use of the divergencefree condition of the magnetic field to constrain the solution and hopefully reduce the null space. Given a known variation for $K(\boldsymbol{r})$, the null space fields $\boldsymbol{B}_{\mathrm{NS}}$ satisfy $K \cdot \boldsymbol{B}_{\mathrm{NS}}=\nabla \phi$. We rewrite this as $\nabla \times\left(K \boldsymbol{B}_{\mathrm{NS}}\right)=0$ or

$\boldsymbol{B}_{\mathrm{NS}} \times \nabla(\ln K)=\boldsymbol{J}_{\mathrm{NS}}, \quad \nabla \cdot \boldsymbol{B}_{\mathrm{NS}}=0$,

where $\boldsymbol{J}_{\mathrm{NS}}$ is the associated current density. We speculate that the additional restriction to force-free fields for which $\boldsymbol{J}$ and $\boldsymbol{B}$ are parallel may make this part of the null space vanish. However, in this paper we restrict ourselves to enforcing $\nabla \cdot \boldsymbol{B}=0$.

To which extent we can reconstruct the divergence-free part $\boldsymbol{V}^{\prime}$ in (9) depends on the geometry of the view directions available. Schuster (2001) has shown that $\boldsymbol{V}^{\prime}$ can be reconstructed from observations from all three space directions. In our case, we have to expect that the solvability is limited by the fact that we usually have observations only from the ecliptic plane and in addition even from the ecliptic plane certain directions onto a given coronal volume element are missing due to the occultation of the Sun.

\section{The inversion approach}

From the discussion of the previous chapter it is clear that the constraint $\nabla \cdot \boldsymbol{B}=0$ should be incorporated into the inversion procedure in order to stabilize the inversion. Therefore, we tried to solve the problem by minimizing the function

$$
\begin{aligned}
F(\boldsymbol{B}) & =\mu \sum_{p, i} w_{p}\left(V_{p, i}^{\text {obs }}-V_{p, i}(\boldsymbol{B})\right)^{2}+\int_{\text {corona }}(\nabla \cdot \boldsymbol{B})^{2} \mathrm{~d}^{3} x \\
& =\mu F_{\text {tomo }}(\boldsymbol{B})+F_{\mathrm{divB}}(\boldsymbol{B})
\end{aligned}
$$

where $V_{p, i}(\boldsymbol{B})$ is the theoretically expected Stokes- $V$ signals (6) in pixel $p$ of image $i$ for an assumed magnetic field distribution $\boldsymbol{B}(\boldsymbol{r})$ and $V_{p, i}^{\mathrm{obs}}$ are the corresponding observed data. The discrepancies between the observed and calculated Stokes- $V$ signal in different pixels $p$ of each image are weighted according to a weight $w_{\mathrm{p}}$ to be discussed below.

Second term, $F_{\text {divB }}$, in (12) is treated like a regularization constraint (Tikhonov \& Arsenin 1977). It was added because the first term $F_{\text {tomo }}$ insufficiently constrains the divergence of $\boldsymbol{B}$. The calculation of this term in (12) allows us to incorporate magnetic field boundary conditions into the inversion procedure. For real observations, the radial magnetic field component observed in the photosphere could serve as boundary condition on the inner surface of the corona. Usually, the regularization term is weighted with a regularization parameter. Instead we multiply the tomography term, $F_{\text {tomo }}$, with parameter $\mu$.

Our ultimate goal is to bring both terms in (12) to zero by varying the magnetic field $\boldsymbol{B}$. The field thus obtained would then comply with the coronal Zeeman observations, it would be divergence free and, if the inner coronal boundary condition is included in the calculation of $\nabla \cdot \boldsymbol{B}$, it would also comply with the photospheric field observations. As a minimization algorithm we will use an iterative Krylov scheme, e.g., conjugate gradients, which has the additional advantage that it minimizes the energy $\int\left(\boldsymbol{B}-\boldsymbol{B}^{(0)}\right)^{2} \mathrm{~d}^{3} x$ of the different magnetic field relative to the initial field $\boldsymbol{B}^{(0)}$ from which the iteration is started. Hence, if we use (12) without any tomography data or with $\mu \rightarrow 0$ we expect to obtain the potential field which complies with the observed photospheric observations if we start the iteration with $\boldsymbol{B}^{(0)}=0$. We use the potential field approximation as starting point for our iterations. According to the structures it is sensitive to any coronal Zeeman observation included in (12) will drive the resulting coronal magnetic field away from the potential field towards the real coronal field configuration.

The weighting function $w_{p}$ in (12) is employed to balance the signals received in different parts of the Zeeman images. Due to the very strong radial decrease of the electron density, the signal on LOS at a great distance from the Sun is much weaker than close to the Sun. From (6), we can roughly estimate this effect by

$V_{p, i} \sim \frac{B_{\mathrm{avr}}}{4 \pi R^{2}} \int_{\operatorname{LOS}_{p, i}} K(\boldsymbol{r}) \mathrm{d} \ell$

where $B_{\text {avr }}$ is the average coronal magnetic field strength. To increase the contribution of signals from distant lines-of-sight and to stabilize the numerical reconstruction of $\boldsymbol{B}$ at larger distances from the Sun, a weight function

$w_{p} \propto \frac{1}{\int_{\operatorname{LOS}_{p, i}} K(\boldsymbol{r}) \mathrm{d} \ell}$

would be appropriate. For the conjugate gradient iteration this weighting effectively acts like a Jakobi precondition. However in practice, in case of real noisy data, the weight function (14) should be modified by setting it's value to zero when signal in LOS measurements of the kernel function is about of a noise level. 

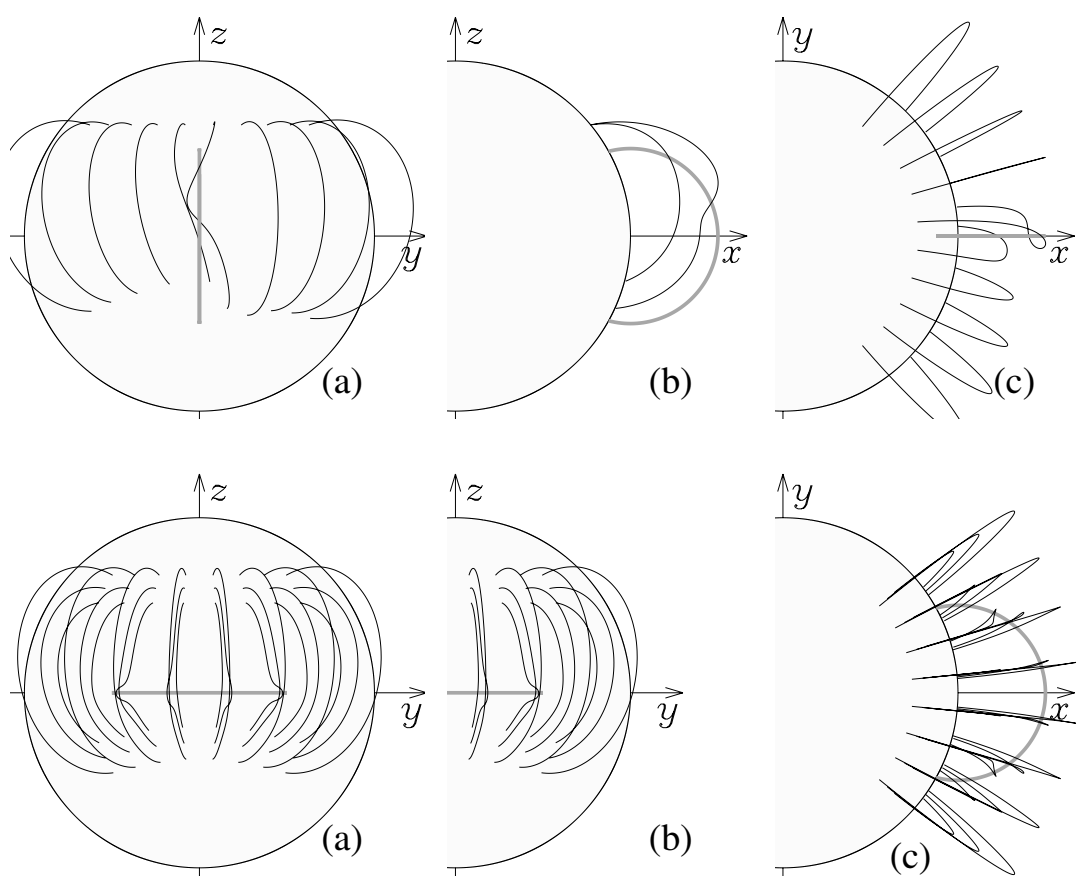

(b)

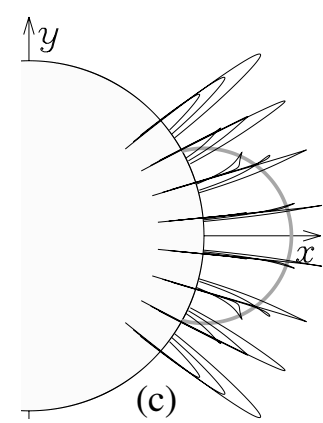

Fig. 2. Model I. Panel a) shows the view from the $x=$ $+\infty$ direction, panel b) shows the view from the $y=-\infty$ direction, and panel c) shows the view from the $z=+\infty$ direction. The perturbing current loop is shown by the thick grey lines.

\section{Numerical setup of the test calculations}

In order to test our inversion approach, we applied it to simulated data from artificial magnetic field configurations. In a successful test, the field configuration should be retrieved from the artificial data.

\subsection{Magnetic field configuration for the test calculations}

We used two model field configurations to test the possibility of the reconstruction. Both models consist of a main dipole field and a non-potential perturbation. In both cases, the axis of the main dipole is inclined in the $x-z$ plane by $10^{\circ}$ with respect to the Sun's rotation axis ( $z$-axis). In the first model (Model I) an additional perturbation due to a circular current in the meridian $x-z$-plane with radius $R_{\odot} / 2$ is superposed onto the dipole. The center of the circular current is located at $\left(R_{\odot} / 2 ; 0 ; 0\right)$ inside the solar sphere (Fig. 2). In the second model (Model II) the perturbation is again due to a circular current which, however, is confined in the equatorial $x-y$-plane. The radius of the current loop and its center are again $R_{\odot} / 2$ and at $\left(R_{\odot} / 2 ; 0 ; 0\right)$, respectively (Fig. 3).

The non-potential field perturbations on the Sun's surface due to these current loops are small and hence we presume that these perturbations are difficult to be reconstructed purely by extrapolation methods from the surface data. The reconstruction of this perturbations from tomography data therefore represents a critical test of our method.

The field lines in the perturbed region (Model I) are twisted, and as seen from the top some of the field lines display an $\mathrm{S}$-shape (Fig. 2a). This deformation is very similar to observed sigmoid loops.

From these test fields the Zeeman data and also the radial magnetic surface field data were calculated as input for our inversion procedure by a simple forward step. Random noise was added to the Zeeman and surface magnetic field data by multiplying the exact data with $(1+\delta)$, where $\delta$ is a uniformly distributed random number in the range $\left(-\epsilon_{\mathrm{n}},+\epsilon_{\mathrm{n}}\right)$ with $\epsilon_{\mathrm{n}}=0.05$.

\subsection{Model for the kernel function $K(r)$}

In the case of Faraday-effect data, the kernel $K(\boldsymbol{r})$ is simply the electron density. In the case of Zeeman-effect data, $K(\boldsymbol{r})$ is defined by (7) from the magnetograph formula (3) if the effect of a finite alignment factor is neglected. The radial dependence of $K(r)$ in this case is largely due to the population density $N_{\alpha_{0} J_{1}}$. It can be related to the coronal electron density $n_{\mathrm{e}}$ by

$N_{\alpha_{0} J_{1}}=\frac{N_{\alpha_{0} J_{1}}}{N_{\text {Ion }}} \frac{N_{\text {Ion }}}{n_{\mathrm{e}}} n_{\mathrm{e}}$

where the first term on the right and side is the excitation ratio of the ion and the second term its abundance ratio. Both these terms may depend on the local solar radiation intensity and on the ambient electron temperature. In our test calculations for simplicity we assume both these terms to be constant and that the density varies only with radial distance from the Sun according to the electron density model by Newkirk (1970). Hence

$$
\begin{aligned}
K(\boldsymbol{r}) \propto & n_{\mathrm{e}}(r) \propto\left[755\left(\frac{r}{R_{\odot}}\right)^{-5.353}-168\left(\frac{r}{R_{\odot}}\right)^{-14.738}\right. \\
& \left.+103800\left(\frac{r}{R_{\odot}}\right)^{-20.446}\right] .
\end{aligned}
$$

Also, instead of (14) we use a slightly different weight function which can be calculated more faster but serves the same purpose as (14):

$w_{p}=\frac{r_{p}^{4}}{n_{\mathrm{e}}\left(r_{p}\right)}$,

where $r_{p}=\sqrt{x_{p}^{2}+y_{p}^{2}}$ is the projected distance of the line-ofsight from image pixel $p$ to the center of the Sun (see Fig. 1).

\subsection{Discretization}

In our test simulations we represent the corona by a spherical shell with inner and outer radial boundaries at $R_{\odot}$ and $2 R_{\odot}$, respectively. The magnetic field is determined at the centers of 
square grid cells with a grid size of $40 \times 40 \times 40$. Hence the grid size is $h=0.1 R_{\odot}$. Similarly, our Zeeman images have $40 \times 40$ pixels with an equivalent grid size of $0.1 R_{\odot}$. The number of pixels is therefore less (in twice) than the optimal number needed to reconstruct structures with size about $0.1 R_{\odot}$ (Zidowitz 1997).

The LOS integrals in (12) are evaluated by line integrals of the linearly interpolated magnetic field values. The line of integration is defined by the projection of the respective pixel center along the line of sight. The integration error introduced in this way is $O\left(h^{3}\right)$.

All rays from the same image are assumed to be parallel as in affine projections as shown in Fig. 1. Hence we neglect the divergence of the observing rays from different pixels which is of the order of $10^{-2}$ if the observations are made from about $1 \mathrm{AU}$ distance from the Sun.

The observations are assumed to be made at a time when the Sun's rotation axis is perpendicular to the view direction $\left(\theta_{\mathrm{LOS}}=\pi / 2\right.$ in the Fig. 1$)$. We associate the $z$-axis of our coronal coordinate system with the Sun's rotation axis. All lines of sight then are parallel to the $x-y$ plane and from the Zeeman effect observations we do not have any information about $z$-component of the magnetic field vector. Observations are assumed to have been made every $10^{\circ}$ for half a solar rotation. The interval in $\sim 10^{\circ}$ could be practically reached by having 1-2 ground based observations per day. However, this interval does not allow us to reconstruct patterns with scale equals to the chosen grid size $\left(0.1 R_{\odot}\right)$ from only tomography data (Zidowitz 1997$)$. We hope that $\nabla \cdot \boldsymbol{B}=0$ condition introduced as regularization constraint in the minimization function (12) allows to overcome this resolution limit.

The discretization of the divergence term is obtained assuming that $\nabla \cdot \boldsymbol{B}$ is a constant inside every grid cell. Its value can then be calculated from $\sum_{\text {faces } s} \Phi_{i, s} / v_{i}$ where $\Phi_{i, s}$ is the magnetic flux out of grid cell $i$ through its face $s$ and $v_{i}$ is the volume of the cell. Then

$$
\int_{\text {corona }}(\nabla \cdot \boldsymbol{B})^{2} \mathrm{~d}^{3} x \simeq \sum_{\text {cells } i} \frac{1}{v_{i}}\left(\sum_{\text {faces } s} \Phi_{i, s}\right)^{2} .
$$

The discretization error obtained this way is again $O\left(h^{3}\right)$ and is comparable to the discretization error of the LOS integration.

\section{Results of the test calculations}

With ideal data one might expect to minimize $F(\boldsymbol{B})$ in (12) to zero in which case the minimizing field $\boldsymbol{B}$ would not depend on the parameter $\mu$ introduced in (12). For real data contaminated with noise the minimization of $F$ below a characteristic value which depends on the noise level is not meaningful because then the reconstructed $\boldsymbol{B}$ starts to be fitted to the noise in the observations (Mozorov's criterion). For ill-conditioned problems, such as the one we deal with here, this results in a strongly amplified noise in the reconstruction.

For our test calculations, the characteristic noise value for the tomography term $F_{\text {tomo }}$ is approximately given by

$\mathcal{E}_{\text {tomo }}=\sum_{p, i} w_{\mathrm{p}}\left(V_{p, i}^{\mathrm{obs}}-V_{p, i}\left(\boldsymbol{B}_{0}\right)\right)^{2} \simeq 0.5 \epsilon_{\mathrm{n}}^{2} \sum_{p, i} w_{\mathrm{p}} V_{p, i}^{2}\left(\boldsymbol{B}_{0}\right)$,

where $\boldsymbol{B}_{0}$ is the model field to be reconstructed. For the divergence term $F_{\text {divB }}$ we use as a characteristic noise estimate the finite divergence which results in (18) if the magnetic flux values $\Phi_{i, s}$ through the solar boundary cell surfaces are perturbed by noise while the field values in the interior of the corona remain unperturbed. Then

$$
\begin{aligned}
\mathcal{E}_{\mathrm{divB}} & \simeq \sum_{\text {boundarycells } i} \frac{1}{v_{i}} \delta \Phi_{i, \text { boundaryface }}^{2} \\
& \simeq 0.5 \epsilon_{\mathrm{n}}^{2} \sum_{\text {boundarycells } i} \frac{1}{v_{i}} \Phi_{i, \text { boundaryface }}^{2}
\end{aligned}
$$

The two parameters which control that $F_{\text {tomo }}$ and $F_{\text {divB }}$ reach about the levels $\mathcal{E}_{\text {tomo }}$ and $\mathcal{E}_{\mathrm{divB}}$, respectively, during the minimization are the regularization parameter $\mu$ in (12) and the number of iteration steps performed.

Conventionally, minimization problems comparable to (12) are analyzed in a diagram which shows the limiting values of $\ln F_{\text {tomo }}$ versus $\ln F_{\text {divB }}$ for various values of the regularization parameter. The optimal values of $\mu$ are then determined by means of the L-curve method (Hansen \& O'Leary 1993). In our case we have to proceed differently because firstly the divergence is not a proper regularization operator due to its large nullspace and secondly the noise level $\mathcal{E}_{\text {tomo }}$ turned out to be much larger than the value of $F_{\text {tomo }}$ at the inflection point of the $\mathrm{L}$ in the L-curve diagram. In this case the value of $\mu$ found from the L-curve analysis is not optimal (Hansen 1993; Hanke 1996).

Figure 4a shows the evolution of the logarithm of the data error, $F_{\text {tomo }}$, versus the logarithm of the divergence term, $F_{\text {divB }}$, during iterations for different values of $\mu$, starting from a potential field solution. The noise levels $\ln \mathcal{E}_{\text {tomo }}$ and $\ln \mathcal{E}_{\text {divB }}$ are marked by dashed lines in the diagram. For values of $\mu$ in the range from $10^{-5}$ to $10^{5}$ we performed more than 5000 iteration steps with the result that in all cases the final value of $F_{\text {tomo }}$ was about a factor 3 below the data noise level $\mathcal{E}_{\text {tomo. }}$. For an optimal reconstruction we could stop the iteration already after approximately 50 iterations when $F_{\text {tomo }}$ just fell below $\mathcal{E}_{\text {tomo }}$. For $\mu$ slightly above $10^{-3}, F_{\text {divB }}$ would at the same time just have crossed $\mathcal{E}_{\text {divB }}$.

The reconstructed three-dimensional magnetic field over a large part of the corona corresponds to the original dipole field. In Fig. 5 we show for the model I the equatorial cross section of the reconstructed field through the perturbing current loop. The current loop intersects the cross section plane in the normal direction. Since also the dipole field is more or less normal to the equatorial plane (the dipole axis is tilted by $10^{\circ}$ ), the field perturbation can clearly be seen in panel (a) which displays the cross section for the original field from which the inversion data was calculated. In panel (b) the same cross section is shown for the potential field reconstruction, while panels (c) and (d) display the reconstruction results for $\mu=10^{-3}$ after 47 and 99 iterations, respectively. Clearly, the main features of the current loop are resolved in the reconstruction but not, as expected, in the potential field.

A problem with our model is that the region perturbed by the current loop fills only a small portion of the coronal volume. Both, $F_{\text {tomo }}$ and $\mathcal{E}_{\text {tomo }}$ are however integrals over the whole corona. Therefore, the reconstruction obtained on the relative magnitudes of $F_{\text {tomo }}$ and $\mathcal{E}_{\text {tomo }}$ may not be optimal for the perturbed region. In a second reconstruction attempt, we therefore seek the optimal $\mu$ and the stopping condition from $F_{\text {tomo }}^{\prime}$ and $\mathcal{E}_{\text {tomo }}^{\prime}$ which differ from the respective prior expressions in that they only include those rays which pass through the region where the magnetic field is markedly perturbed by the current loop. We define the perturbed region by the conditions $x>0$, $x \tan \left( \pm \frac{\pi}{18}\right)<y<x \tan \left( \pm \frac{\pi}{18}\right), R_{\odot}^{2}<x^{2}+y^{2}+z^{2}<\left(2 R_{\odot}\right)^{2}$, and $-R_{\odot}<2 z<R_{\odot}$. 


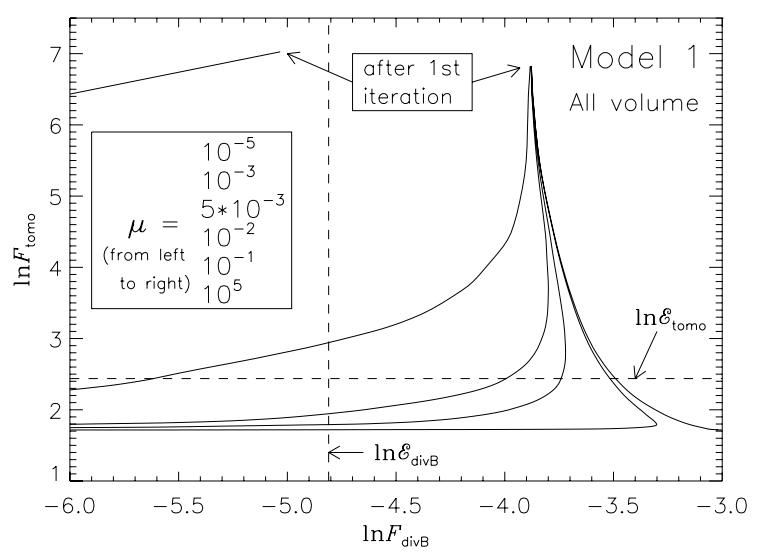

(a)

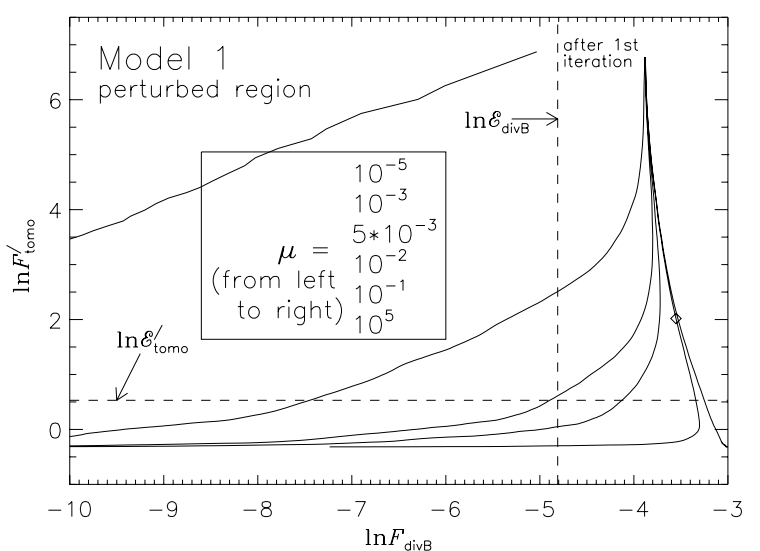

(b)

Fig. 4. The evolution of the logarithm of the data error $F_{\text {tomo }}$ versus the logarithm of the divergence term $F_{\text {divB }}$ during the iterations for different values of $\mu$. The numbers near the lines are the number of iterations which were done to reach the data noise level $\mathcal{E}_{\text {tomo }}$ (dashed horizontal line).
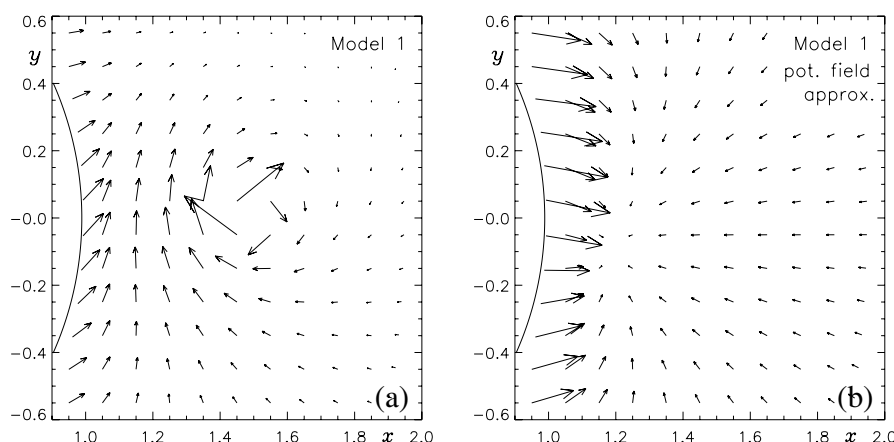
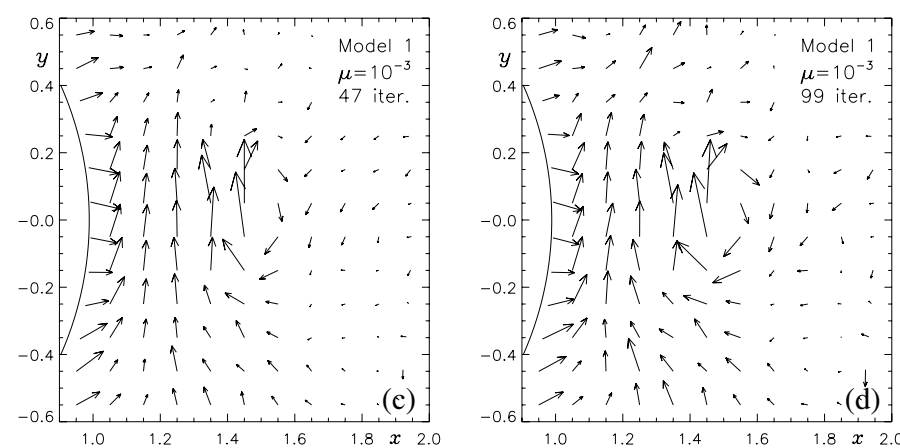

Fig. 5. Model I, the cross-section through the $z=0.05$ plane: original field a), potential field approximation b), tomographic reconstruction for $\mu=10^{-3}$ obtained after 47 iterations $\mathbf{c}$ ) and after 99 iterations d).

Figure $4 \mathrm{~b}$ shows the decrease of $\ln F_{\text {tomo }}^{\prime}$ and $\ln F_{\text {divB }}$ for these calculations for iterations with the different values of $\mu$. Again, the value of the $\mathcal{E}_{\text {tomo }}^{\prime}$ is marked by a dashed horizontal line in Fig. 4b. To reach the noise level $\mathcal{E}_{\text {tomo }}^{\prime}$ for $F_{\text {tomo }}^{\prime}$, it is now necessary to perform about 100 iterations, the optimal regularization parameter again is about $\mu=10^{-3}$. Figure $5 \mathrm{~d}$ shows the reconstruction obtained for $\mu=10^{-3}$ after 99 iterations. The perturbed region now is reconstructed indeed better than in the previous case after only 47 iterations. The stopping criterion therefore is not strict: the more iteration steps were performed, the more small scale features were included into the reconstructed magnetic field. Together with these small scales the noise also grows until it eventually dominates the result. This is not yet the case in Fig. 5d, although the noise in the reconstructed field is indeed larger than in Fig. $5 \mathrm{c}$.

A similar minimization procedure has been performed for the second model using similar rules for the selection of $\mu$ and stopping the iteration. A meridional cross section of the reconstructed magnetic field of the second model is shown in Fig. 6. Again the current loop, now in the equatorial plane, intersects the cross section plane in normal direction. Since the field perturbation is superposed on the background dipole field, the perturbation can only marginally be seen even in the original field displayed in panel a.

In the reconstructions shown in panels $\mathrm{c}$ and $\mathrm{d}$ which take into account the Zeeman data the field perturbations are absent. The Zeeman-effect observations seem to be insensitive to current loops in a plane normal to the vertical axis. As a first, intuitive explanation of this result we may take the fact that the perturbed magnetic field lies more or less in the meridional planes while the longitudinal Zeeman effect is to lowest order sensitive to the field component normal to these planes. For a more precise argument we have to recall that all view directions lie in $x-y$ planes and the field perturbation of a current loop projected onto these planes is almost irrotational. In agreement with the basic features of vector tomography stated in Sect. 3 the irrotational component of the vector field cannot be reconstructed from this type of measurements. Even the regularization by the divergence term does not help because the tomography data involves only $x$ - and $y$-components of the field, but the divergence term involves all three components. The reconstruction would be possible if we had information about the $z$-component of the field. These may be obtained from coronal Hanle-effect measurements. A less realizable alternative would be out-of-ecliptic Zeeman observations which are also sensitive to the $z$-component.

\section{Discussion}

The goal of the paper is to investigate the possibility of using the observations of the longitudinal Zeeman effect in coronal emission lines for a reconstruction of non-potential features of the coronal magnetic field by means of a tomographic inversion.

The reconstruction of vector fields by tomographic means is much more difficult than to reconstruct scalar fields. The LOS integrals of LOS field components, as obtained from longitudinal Zeeman data, are known to be insensitive to the rotation-free 

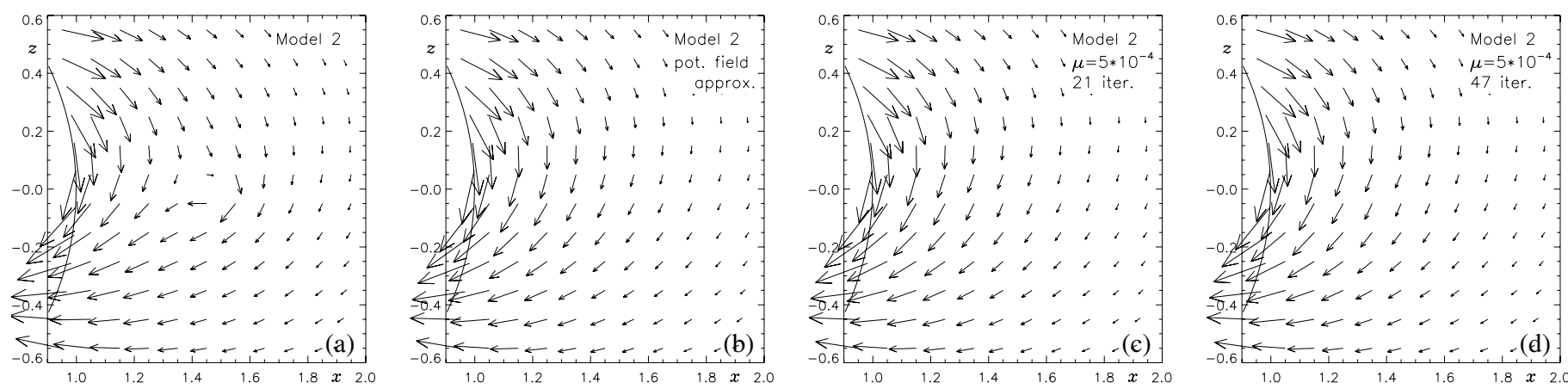

Fig. 6. Model II, the cross-section by $y=0.05$ plane: original field a), potential field approximation $\mathbf{b}$ ), tomographic reconstruction for $\mu=5 \times 10^{-4}$ obtained after 21 iterations c) and after 47 iterations d).

part of the field. In order to limit these deficiencies we combined the inversion scheme with the requirement to produce a divergence-free field. In this way the observed normal magnetic field component at the solar surface can be included into the inversion procedure with the result that the scalar potential which is invisible in the longitudinal Zeeman data is uniquely specified.

The approach bears some similarity with the procedure suggested by Wheatland et al. (2000) to extrapolate force-free fields from photospheric field data. In their scheme the tomography data term $F_{\text {tomo }}$ is replaced by $\int|\boldsymbol{J} \times \boldsymbol{B}|^{2}$. Currently we are investigating whether the combination of both terms, i.e., the tomographic reconstruction confined to force-free fields yields more stable results. The fact that the tomographic reconstruction requires stationarity of the coronal magnetic field during the observation lends some justification to the assumption that the field is also force-free.

The input data necessary for our inversion are a set of Zeeman-effect observations of a coronal line for at least half a solar rotation and a photospheric synoptic chart obtained from magnetograms recorded over the same time interval. While the latter are regularly produced by ground-based and space-borne instruments, the former observations are difficult to perform. The experimental efforts to observe the magnetically sensitive coronal lines needed for our inversion have, however, increased in recent years. Especially the infrared spectral range is suitable for the detection of the Zeeman-effect under coronal conditions than observations at optical wavelengths. This is due to the fact that Zeeman splitting is proportional to the square of the wavelength, but thermal broadening increases only proportional to the wavelength itself. Moreover, the disturbing scatter of the infrared radiation by the Earth's atmosphere is less than in the optical wavelength range. Recent progress in spectropolarimetric observations of infrared coronal lines (Lin et al. 2000, 2004) gives hope that in the near future data will be available for a reconstruction of the 3D structure of the coronal magnetic field as proposed here.

Since the purpose of the present paper is to find out to which extent the inversion problem is solvable in general, we applied it only to artificially generated data and neglected the alignment factor which does not affect the conditioning of the inversion problem. This may easily be improved when our method is applied to real data. The inclusion of the alignment factor into the inversion comes at the expense of more computational complexity, however.

The assumption about stationarity of the corona is more fundamental to tomography and limits the use of our method to situations with low solar activity. In addition, the spatial resolution of the tomographic inversion is limited to scales $\sim 0.1 R_{\odot}$ close to the solar surface and we expect that $\boldsymbol{B}$ on these scales evolves slower than at smaller scales.

In the present paper, we investigated two illustrative model field configurations. These models comprised a background solar dipole field with an embedded coronal current loop. While the potential field component (essentially the dipole field) can be reproduced from the photospheric surface observations alone, e.g., by use of Green's theorem, it is practically impossible to reconstruct the field perturbations due to the current loops by extrapolation methods. The main test of our calculations therefore was to find out the quality with which these perturbation fields were reproduced.

Good agreement between the model field and our field reconstruction were obtained for the first model where the current loop has north-south orientation. For the second model with a current loop in east-west direction the reconstruction based on Zeeman data failed. We anticipate that fields with an orientation as in the second model can be reconstructed more favorably with the Hanle-effect data which yield information about the magnetic field in the plane of the sky. Detailed results of the corresponding test calculations are deferred to a follow-up paper where we investigate inversions of the Hanle-effect data and their combination with the Zeeman-effect data.

The results presented here are only a first demonstration of the possibilities and limitations of using coronal observations in magnetically sensitive lines to reconstruct the 3D structure of the coronal magnetic field. Our results demonstrate that it is in principle possible to reconstruct at least some non-potential configurations of the coronal magnetic field from coronal Zeeman-effect data. However, some more steps need to be performed before our method can be applied to real data which will be addressed in future publications:

i) Different and more realistic coronal magnetic field configurations, e.g., the field above active regions or more realistic streamer-type field structures should be studied to find out which details of the field can or cannot be reconstructed.

ii) With the code we have developed, we can systematically study in test calculations how much noise is tolerable to achieve a certain precision in the reconstruction. Since the integration time to obtain the longitudinal Zeeman data is about an hour for state-of-the-art instruments (Lin et al. 2004), this information about tolerable data noise levels could be very helpful to limit the integration time.

iii) It is also important to investigate how data gaps reduce the quality of the reconstruction results.

iv) An additional constraint to produce only force-free fields could stabilize the reconstruction and partly compensate for noise or data gaps. The problem which results then is similar 
to the Wheatland algorithm, except that tomography terms are added. Since the Wheatland algorithm together with appropriate boundary values on all outer boundaries is known to yield a unique result, the inclusion of tomography data, even an incomplete data set over a few view directions, could help to eliminate ambiguities which arise from the Wheatland extrapolation due to unknown lateral boundary values.

v) Observations of the Faraday rotation of the linearly polarized radio signals traveling through the corona give information very similar to the longitudinal Zeeman effect. However, the data is obtained at a given time only along a single line of sight rather than on a whole set of lines of sight corresponding to the pixels of an image. It would be interesting to study how useful these sparse measurements are for the reconstruction of the coronal field. An example of coronal Faraday-rotation observations are the measurements of Jensen et al. (2005), who used the radio signal of the Cassini spacecraft during its passage behind the Sun as the radio source.

vi) The present work could be considered as a first step towards a systematic inversion of the observation of all four Stokes parameters from coronal emission lines to obtain a coronal magnetic field model.

Acknowledgements. Many thanks to N.-E. Raouafi and T. Wiegelmann for the helpful discussions during the work. The authors also thank referee A. Lopez for useful comments.

The presented work was supported by the International Max-Planck Research School on Physical Processes in the Solar System and Beyond at the Universities of Braunschweig and Göttingen.

\section{References}

Arnaud, J. 1982, A\&A, 116, 248

Arnaud, J., \& Newkirk, G., Jr. 1987, A\&A, 178, 263

Casini, R., \& Judge, P. G. 1999, ApJ, 522, 524

Casini, R., \& Judge, P. G. 2000, ApJ, 533, 574
Charvin, P. 1965, Ann. Astrophys., 28, 877

Davila, J. M. 1994, ApJ, 423, 871

Davila, J. M., \& Thompson, W. T. 1992, ApJ, 389, 91

Demoulin, P., Cuperman, S., \& Semel, M. 1992, A\&A, 263, 351

House, L. L. 1977, ApJ, 214, 632

House, L. L., Querfeld, C. W., \& Rees, D. E. 1982, ApJ, 255, 753

Jensen, E. A., Bird, M. K., Asmar, S. W., et al. 2005, Adv. Space Res., 36, 1587

Jiao, L., McClymont, A. N., \& Mikić, Z. 1997, Sol. Phys., 174, 311

Judge, P. G. 1998, ApJ, 500, 1009

Landi Degl'Innocenti, E. 1982, Sol. Phys., 79, 291

Landi Degl'Innocenti, E. 1984, Sol. Phys., 91, 1

Landi Degl'Innocenti, E., \& Landolfi, M. 2004, Polarization in spectral lines (Dordrecht: Kluwer Academic Publishers)

Lin, H., Kuhn, J. R., \& Coulter, R. 2004, ApJ, 613, 177

Lin, H., Penn, M. J., \& Tomczyk, S. 2000, ApJ, 541, 83

Natterer, F. 1986, The Mathematics of Computerized Tomography (Chichester: Wiley)

Newkirk, G., Jr., Dupree, R. G., \& Schmahl, E. J. 1970, Sol. Phys., 15, 15

Norton, S. J. 1989, Geophys. J., 97, 161

Osman, N. F., \& Prince, J. L. 1998, Inverse Problems, 14, 185

Querfeld, C. W. 1982, ApJ, 255, 764

Raouafi, N.-E., Sahal-Bréchot, S., \& Lemaire, P. 2002, A\&A, 396, 1019

Sahal-Brechot, S. 1974a, A\&A, 32, 147

Sahal-Brechot, S. 1974b, A\&A, 36, 355

Sahal-Brechot, S. 1977, ApJ, 213, 887

Sakurai, T. 1989, Space Sci. Rev., 51, 11

Schuster, T. 2001, Inverse Problems, 17, 739

Sobelman, I. I. 1972, Introduction to the theory of atomic spectra (Oxford: Pergamon Press)

Sparr, G., \& Strahlen, K. 1998, Vector field tomography, an overview, Technical report, Lund University (Lund Institute of Technology: Center for Mathematical Sciences)

Stelzried, C. T., Levy, G. S., Sato, T., et al. 1970, Sol. Phys., 14, 440

Stenflo, J. O. 1994, Solar magnetic fields (Dordrecht: Kluwer Academic Publishers)

Tikhonov, A. N., \& Arsenin, V. A. 1977, Solutions of Ill-posed Problems (Washington: Winston \& Sons)

Thorne, A., Litzen, U., \& Johansson, S. 1999, Spectrophysics: Principles and Applications (Berlin: Springer)

Wheatland, M. S., Sturrock, P. A., \& Roumeliotis, G. 2000, ApJ, 540, 1150

Wiegelmann, T., Lagg, A., Solanki, S. K., Inhester, B., \& Woch, J. 2005, A\&A, 433, 701

Zidowitz, S. 1997, Ph.D. Thesis, TU-Braunschweig/Max-Planck-Institut f. Aeronomie, MPAE-W-100-97-16

Zidowitz, S. 1999, JGR, 104, 9727 